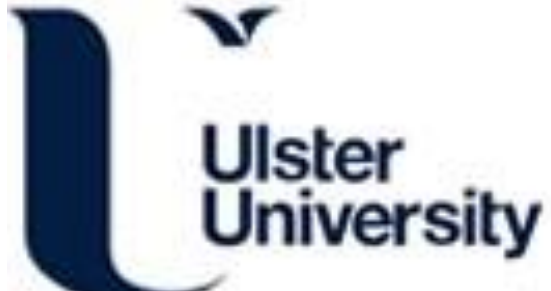

\section{A Usability Study of a Critical Man-Machine Interface: Can Layperson Responders Perform Optimal Compression Rates When Using a Public Access Defibrillator with Automated Real-Time Feedback During Cardiopulmonary Resuscitation?}

Torney, H., O’Hare, P., Davis, L., Delafont, B., Bond, R. R., McReynolds, H., McLister, A., McCartney, B., Di Maio, R., \& McEneaney, D. (2016). A Usability Study of a Critical Man-Machine Interface: Can Layperson Responders Perform Optimal Compression Rates When Using a Public Access Defibrillator with Automated Real-Time Feedback During Cardiopulmonary Resuscitation? IEEE Transactions on Human-Machine Systems, 46(5), 749-754. https://doi.org/10.1109/THMS.2016.2561267

Link to publication record in Ulster University Research Portal

Published in:

IEEE Transactions on Human-Machine Systems

Publication Status:

Published online: 10/06/2016

DOI:

10.1109/THMS.2016.2561267

Document Version

Author Accepted version

\section{General rights}

Copyright for the publications made accessible via Ulster University's Research Portal is retained by the author(s) and / or other copyright owners and it is a condition of accessing these publications that users recognise and abide by the legal requirements associated with these rights.

\section{Take down policy}

The Research Portal is Ulster University's institutional repository that provides access to Ulster's research outputs. Every effort has been made to ensure that content in the Research Portal does not infringe any person's rights, or applicable UK laws. If you discover content in the Research Portal that you believe breaches copyright or violates any law, please contact pure-support@ulster.ac.uk. 


\title{
A Usability Study of a Critical Man-Machine Interface: Can Layperson Responders Perform Optimal Compression-Rates when using a Public Access Defibrillator with Automated Real-Time Feedback during Cardiopulmonary Resuscitation?
}

\author{
Hannah Torney, Peter O’Hare, Laura Davis, Bruno Delafont, Raymond Bond, Hannah McReynolds, \\ Anna McLister, Ben McCartney, Rebecca Di Maio, and David McEneaney
}

\begin{abstract}
Objective: Many public access defibrillators (PAD) incorporate computer programs to provide audio-visual feedback to assist the user to deliver cardiopulmonary resuscitation (CPR) according to current international guidelines. This usability study assessed if a PAD integrated with a real-time audio-visual CPR feedback system can guide lay-users to optimum chest compression rates, and if it is detrimental to chest compression depth. Methods: Randomly selected volunteers $(15+$ years) were recruited for two experiments. Experiment $1(n=156)$ assessed the time taken to achieve the "Good speed" audio prompt (i.e. perform compressions at a rate of 100-120 compressions per minute) and chest compression fraction (CCF). Experiment 2 $(n=140)$ assessed the effect of rate-only CPR feedback on chest compression depth. Two devices of the same model were used, one with CPR rate feedback, and the other with CPR feedback disabled. The difference in compression depths and CCF were assessed. Results: Experiment 1: A total of 136 (87.2\%) participants achieved "Good speed" within 45 seconds with a mean CCF of $90.3 \%$ recorded. Experiment 2: The device with feedback lead to a mean (SE) depth of $24.61 \mathrm{~mm}(0.99)$ compared to $20.08 \mathrm{~mm}(0.96)$ for the feedback disabled device. ANCOVA analysis provided a mean significant difference (Standard Error; SE) of $4.52 \mathrm{~mm}(1.38 \mathrm{~mm}$; $p$-value $=0.001)$ favouring the device with CPR rate feedback. Conclusions: CPR rate-only feedback was not detrimental to chest compression depth and suggests rate-only feedback may improve compression depth. Significance: The incorporation of clear, intuitive, audio-visual CPR feedback systems can assist lay-users to optimise compression rates and maintain a high $\mathrm{CCF}$.
\end{abstract}

Index Terms - Public Access Defibrillators; Cardiopulmonary Resuscitation (CPR); Usability

Submitted for review on 26 June 2015

H. Torney, P. O’Hare, L. Davis, H. McReynolds, A. McLister, B. McCartney and R. Di Maio are with HeartSine Technologies Ltd, Belfast. H. McReynolds, A. McLister, B. McCartney and R.B. Bond are with Ulster University, Newtownabbey. B. Delafont is with Exploristics Ltd, Belfast. D. McEneaney is with Craigavon Area Hospital, Portadown. Corresponding author email: hannah.torney@heartsine.com

\section{INTRODUCTION}

$\mathrm{O}$ ut-of-hospital cardiac arrest (OHCA) accounts for 300,000 and 420,000 deaths per year in Europe and the US respectively [1] [2]. Effective cardiopulmonary resuscitation (CPR) coupled with early defibrillation by an automated external defibrillator (AED) has been shown to significantly enhance OHCA survival rates [3] [4] [5]. International guidelines recommend that in order to perform efficacious CPR, chest compressions must be administered at a rate of 100-120 compressions per minute (CPM). Suboptimal chest compression rates have been shown to directly compromise haemodynamics and perfusion of vital organs [6]. Chest compression fraction (CCF), the proportion of time that chest compressions are performed during a cardiac arrest event, should be prioritised over compression depth, chest recoil and ventilations. A CCF of $80 \%$ should be achievable in a variety of settings, according to a 2013 American Heart Association consensus statement [7].

Despite efforts to train members of the public in basic life support (BLS), CPR is still performed inadequately by the majority of lay responders [8] [9]. Layperson CPR skill retention rates have been reported to decline in as little as two months [10]. The provision of real-time audio CPR feedback could address shortfalls in training and help maintain BLS skills [11], thus reducing the need for repeated training.

Many public access defibrillators incorporate real-time feedback mechanisms to help rescuers deliver CPR according to current guidelines.

A usability study comprising of two experimental phases was devised and is presented herein. As compression rate is a key metric of effective CPR, Experiment 1 assessed if an AED integrated with automated real-time CPR feedback can help guide lay-rescuers to an optimal chest compression rate during a simulated resuscitation attempt. Following this, Experiment 2 assessed if real-time rate-only CPR feedback was detrimental to the applied depth of chest compressions when compared to an equivalent device with $\mathrm{CPR}$ rate 
feedback disabled.

\section{METHODS AND MATERIALS}

\section{A. Experiment 1 -Effect of real-time CPR feedback on chest compression rate}

Randomly selected male and female volunteers, aged at least 15 years, were recruited in a shopping mall. Each participant was given background information to the study and asked to complete a Pre-Test questionnaire to capture demographical information and any previous CPR and/or AED training. This study was designed to assess the ability of minimally trained users to interact with an audio-visual CPRrate feedback interface, and thus, those with advanced medical training or training on any HeartSine AEDs were excluded. Those trained to use other defibrillators could participate. Prior to commencement of the testing phase, a manikin clothed in a front opening sweater was placed on the floor of the test room (where the manikin represented a female, it was dressed with a bra underneath the sweater).

A HeartSine samaritan ${ }^{\circledR}$ PAD 450P (SAM 450P) public access defibrillator was used for this study. The shock feature was disabled by removing the charging resistor, preventing the device from physically delivering a shock, ensuring participant safety. This device can record the impedance cardiogram (ICG) via the defibrillator pads, enabling detection of the chest compressions generated as a result of the change in the physical impact on the thorax. The rescuer receives real-time audio feedback instructing the user to "Push faster", "Push slower" or that compressions are at a "Good speed". Additionally, the interface has a sequence of light emitting diodes (LEDs) indicating the user's compression rate (Fig. 1). A vital signs manikin capable of simulating a range of ECG and ICG waveforms was used to emulate patient physiology (Fig. 2).

\begin{tabular}{cccc}
\hline CPR Speed & $\begin{array}{c}\text { Corresponding Voice } \\
\text { Prompt }\end{array}$ & LED Lights Displayed & $\begin{array}{c}\text { Corresponding } \\
\text { CPR Chest } \\
\text { Compression } \\
\text { Rate (CPM) }\end{array}$ \\
\hline Too Slow & "Push Faster" & & $<90$ \\
\cline { 3 - 4 } & Good \\
Compressions & "Good Speed" & & $90-100$ \\
\hline Too Fast & "Push Slower" & & $100-120$ \\
\hline
\end{tabular}

Fig. 1. The sequence of operation of voice prompts and LED indicator status over a range of chest compression rates.

Before entering the test room, the participant was instructed that they would find a simulated cardiac arrest victim lying on the floor. They were told that an AED would be placed next to the victim, which can be used to deliver an electrical shock to the victim's heart to help it beat as normal. They were informed that they will be asked to perform CPR, but no information or instruction on how to do so was provided. Each participant was advised to treat this as an emergency situation and act with urgency, but that there is no need to "Call for medical assistance" as instructed by the device.

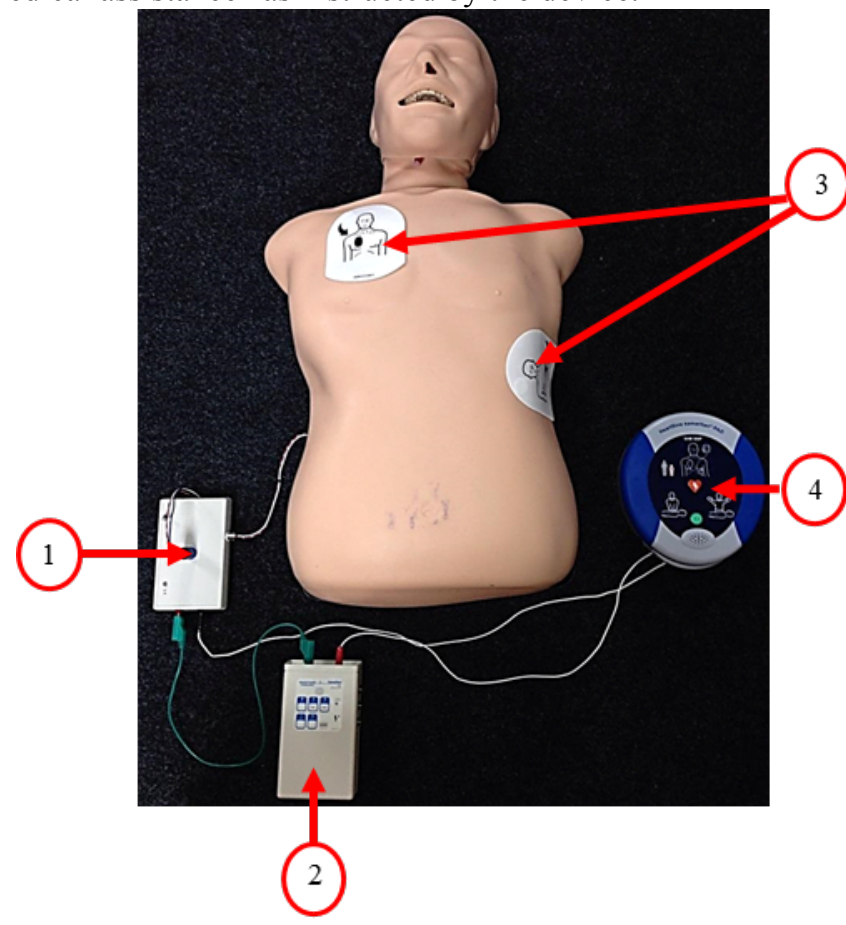

1. Vital signs manikin interface module

2. Vital signs manikin ECG waveform control box

3. Pad-placement. The pads are not physically connected to the device, but are stored and removed from the device in a similar manner to the shock enabled device

4. SAM 450P device. The device is connected to the vital signs manikin directly through the electrodes

Fig. 2. Experimental set-up of the test device and vital signs manikin

The participant was asked to turn on the defibrillator and follow the audio prompts. They were required to remove the patient's clothing, correctly position the electrode pads on the manikin and deliver a shock. Although the shock feature was disabled, the device software still enabled the device to behave as though a shock was delivered.

After shock delivery, the participant was required to perform CPR for 2 minutes. The participant's actions were video recorded during the test and observed by an investigator for qualitative and quantitative data collection. No communication between the investigator and the participant was permitted during the test. Upon completion, the participant was asked to complete a post-test questionnaire to gauge user opinion on the usability of the device and its user interface.

\section{Measurements}

The primary dependent variable assessed was the proportion of participants who achieved "Good speed" (i.e. achieved a compression rate of 100-120 CPM) within 45 seconds of the device instructing the user to "Begin CPR". CCF for the 2 minute CPR cycle was calculated. Variables such as time-tofirst-shock and time-to-pad-placement were also stratified by age group, and CPR and defibrillator experience. 
The secondary dependent variable assessed was the time-tofirst-shock. This was measured from the moment the user was instructed to "Turn on the device" to time of cardioversion. The median time-to-first-shock was derived from KaplanMeier estimates with a $95 \%$ confidence interval (CI). The time-to-first-shock was to be censored if participants did not manage to perform a shock within 10 minutes. The $95 \%$ confidence limits around the median follow the computation described by Kalbfleisch and Prentice [12]. A log-rank test was used to assess the difference in time-to-first-shock between demographical groups.

\section{B. Experiment 2-Effect of real-time CPR rate-only feedback on compression depth}

The second experiment was to determine if rate-only CPR feedback has a detrimental effect on chest compression depth, when compared to a device that does not provide CPR rate feedback. Experiment 2 setup was identical to Experiment 1 setup, with an entirely new set of participants recruited to prevent bias.

Experiment 2 included two SAM 450P defibrillators, the same model as used in Experiment 1. The test device incorporated CPR feedback, whereas CPR rate feedback (both audible prompts and LEDs) was disabled on the control device.

As this experiment focused only on the effect of audiovisual feedback on compression depth, each participant was informed that the electrodes would already be attached and that they would not be expected to deliver a shock. They were instructed to turn on the device and follow the audio instructions, as per the previous experimental set-up. No instructions on how to perform CPR were provided.

Participants were randomly assigned a test device or control device, which was directly connected to the vital signs manikin through the electrode leads, enabling detection of the simulated ICG signals from the manikin.

\section{Measurements}

The primary dependent variable in this study was the mean amplitude of the displacement distance of the potentiometer over the two minute CPR period. The estimated difference in compression depth between devices was derived using analysis of covariance (ANCOVA) adjusted for age and gender, with a $95 \%$ CI. The secondary dependent variables included the proportion of participants who achieved "Good speed" within 45 seconds, and CCF. The difference in the proportion of participants achieving "Good speed" between devices was tested using a Chi-square test.

\section{RESULTS}

\section{A. Experiment 1 Results}

A total of 158 participants were assessed, with two participants excluded due to investigator data recording errors. Demographical information of all test participants $(n=156)$ is presented in Table I.

Of the total 156 test participants recruited, 136 (87.2\%) participants achieved CPR compressions at a "Good speed" within 45 seconds of the voice prompt "Begin CPR". Of the participants who had previous CPR training $(n=83), 73$ $(88.0 \%)$ achieved a good compression rate within 45 seconds. Similarly, of the participants who did not have previous CPR training $(n=73), 63(86.3 \%)$ achieved a good compression rate within 45 seconds. Of these 136 participants, 14 participants did not maintain compressions at this rate.

Twenty-seven (17.3\%) of the total participants were prompted to "Push faster", and $17(10.9 \%)$ of the total participants were prompted to "Push slower".

Of the 20 participants who did not achieve the target rate within 45 seconds, 8 achieved good CPR speed within 1 minute. The lowest CCF of these 20 participants was $82 \%$, which is above the threshold of $80 \%$ recommended in the AHA Consensus Statement [10].

The initial rate of CPR improved for all participants with audio-visual feedback. All participants achieved a "Good speed" voice prompt at least twice during the 2 minutes of CPR (the maximum number of "Good speed" prompts that any one user could achieve is 9).

The median time to reach "Good speed" was 27 seconds for all participants. Approximately half of this test population (46.8\%) had no prior CPR or defibrillation training. The CCF was found to range from $31 \%$ to $98 \%$ and the mean (Standard Deviation; SD) percentage CCF was $90.3 \%( \pm 9.2 \%)$ with a median percentage $\mathrm{CCF}$ of $94.4 \%$. Only 16 (10.1\%) participants had a CCF of less than $80 \%$. The minimum timeto-first-shock recorded was 49 seconds, and the maximum time was 110 seconds.

TABLE I

DEMOGRAPHICAL INFORMATION FOR ALL PARTICIPANTS

\begin{tabular}{|c|c|c|c|c|c|c|c|c|}
\hline & \multicolumn{2}{|c|}{ Gender } & \multicolumn{2}{|c|}{ Age Group (years) } & \multicolumn{2}{|c|}{ Education } & \multicolumn{2}{|c|}{ Prior CPR or Defibrillation Training } \\
\hline \multirow{5}{*}{$\begin{array}{c}\text { Experiment } \\
\quad 1\end{array}$} & Male & $72(46.2 \%)$ & $15-21$ & $28(17.9 \%)$ & Some High School & $9(5.8 \%)$ & None & $73(46.8 \%)$ \\
\hline & Female & $84(53.8 \%)$ & $22-64$ & $101(64.7 \%)$ & High School & $36(23.1 \%)$ & CPR Training only & $61(39.1 \%)$ \\
\hline & & & $65+$ & $27(17.3 \%)$ & Some College & $48(30.8 \%)$ & $\begin{array}{l}\text { CPR and Defibrillator } \\
\text { Training }\end{array}$ & $22(14.1 \%)$ \\
\hline & & & & & College & $47(30.1 \%)$ & & \\
\hline & & & & & Post Graduate & $16(10.3 \%)$ & & \\
\hline \multirow{3}{*}{$\begin{array}{c}\text { Experiment } \\
\quad 2\end{array}$} & Male & $67(47.9 \%)$ & $15-21$ & $31(22.1 \%)$ & Some High School & $31(22.1 \%)$ & None & $80(57.1 \%)$ \\
\hline & Female & $73(52.1 \%)$ & $22-64$ & $84(60.0 \%)$ & High School & $44(31.4 \%)$ & CPR Training only & $34(24.3 \%)$ \\
\hline & & & $65+$ & $25(17.9 \%)$ & Some College & $48(34.3 \%)$ & $\begin{array}{l}\text { CPR and Defibrillator } \\
\text { Training }\end{array}$ & $26(18.6 \%)$ \\
\hline
\end{tabular}


$\begin{array}{ll}\text { College } & 9(6.4 \%) \\ \text { Post Graduate } & 8(5.8 \%)\end{array}$

The estimated median time-to-first-shock was 67.0 seconds, with lower quartile and upper quartiles for time-to-first-shock of 62.5 seconds and 73.0 seconds respectively. Table II shows the time-to-pad-placement and time-to-first-shock according to age group, CPR and defibrillator experience.

The effect of age on time-to-first-shock was confirmed by a significant log-rank test (log-rank statistic $=12.25, \mathrm{df}=2$, $\mathrm{p}=0.002$ ), however, there is no evidence that this difference would be clinically significant. Participants with defibrillator experience showed a significantly lower median time-to-firstshock (64.5 seconds) than participants without such experience $(67.0$ seconds; log-rank statistic $=7.69, \mathrm{df}=1$, $\mathrm{p}=0.006)$.

TABLE II

TIME-TO-PAD-PLACEMENT AND TIME-TO-FIRST SHOCK ACCORDING TO AGE GROUP, CPR AND DEFIBRILLATOR EXPERIENCE

\begin{tabular}{|c|c|c|c|c|}
\hline & $\begin{array}{l}\text { TOTAL } \\
(n=156)\end{array}$ & $\begin{array}{c}15-21 \\
\text { years } \\
(n=28)\end{array}$ & $\begin{array}{c}22-64 \\
\text { years } \\
(n=101)\end{array}$ & $\begin{array}{c}65+\text { years } \\
(n=27)\end{array}$ \\
\hline $\begin{array}{l}\text { Median time-to- } \\
\text { pad placement (s) }\end{array}$ & 48 & 47.5 & 49 & 53 \\
\hline Min, $\operatorname{Max}(\mathrm{s})$ & 34,90 & 36,54 & 34,80 & 35,90 \\
\hline $\begin{array}{l}\text { Median time-to- } \\
\text { first shock (s) }\end{array}$ & 67 & 65 & 67 & 71 \\
\hline Min, Max (s) & 49,110 & 54,79 & 49,108 & 58,110 \\
\hline & \multicolumn{2}{|c|}{$\begin{array}{c}\text { CPR Experience } \\
(n=83)\end{array}$} & \multicolumn{2}{|c|}{$\begin{array}{c}\text { No CPR Experience } \\
(n=73)\end{array}$} \\
\hline $\begin{array}{l}\text { Median time-to- } \\
\text { pad placement (s) }\end{array}$ & \multicolumn{2}{|c|}{48} & \multicolumn{2}{|c|}{49} \\
\hline Min, Max (s) & \multicolumn{2}{|c|}{34,90} & \multicolumn{2}{|c|}{34,73} \\
\hline $\begin{array}{l}\text { Median time-to- } \\
\text { first shock (s) }\end{array}$ & \multicolumn{2}{|c|}{65} & \multicolumn{2}{|c|}{67} \\
\hline Min, Max (s) & \multicolumn{2}{|c|}{49,110} & \multicolumn{2}{|c|}{53,97} \\
\hline & \multicolumn{2}{|c|}{$\begin{array}{c}\text { Defibrillator } \\
\text { Experience }(n=22)\end{array}$} & \multicolumn{2}{|c|}{$\begin{array}{c}\text { No Defibrillator } \\
\text { Experience }(n=134)\end{array}$} \\
\hline $\begin{array}{l}\text { Median time-to- } \\
\text { pad placement (s) }\end{array}$ & \multicolumn{2}{|c|}{46.5} & \multicolumn{2}{|c|}{49} \\
\hline Min, $\operatorname{Max}(s)$ & \multicolumn{2}{|c|}{34,65} & \multicolumn{2}{|c|}{34,90} \\
\hline $\begin{array}{l}\text { Median time-to- } \\
\text { first shock (s) }\end{array}$ & \multicolumn{2}{|c|}{64.5} & \multicolumn{2}{|c|}{67} \\
\hline Min, Max (s) & \multicolumn{2}{|c|}{49,80} & \multicolumn{2}{|c|}{53,110} \\
\hline
\end{tabular}

\section{B. Experiment 2 Results}

In the second experiment, 141 participants were recruited to engage with one of two devices (i.e. the test device [with CPR rate feedback] or the control device [without CPR rate feedback]). One participant was excluded due to insufficient CPR. The demographical information of the test participants is presented in Table I.

The ANCOVA analysis between the two test devices provided a mean difference (Standard Error; SE) of $4.52 \mathrm{~mm}$ $( \pm 1.38)$ with $95 \% \mathrm{CI}=(1.79,7.25)$ for the device with CPR rate feedback when compared to the device without CPR rate feedback (t-value $=3.28, \mathrm{df}=135$, two-sided $p$-value $=0.001$ ). This indicates a significant difference in measured CPR compression depth between the device groups, in favour of the device with CPR rate feedback.

No participants, in either test group, reached over $50 \mathrm{~mm}$ compression depths, as is recommended by the AHA and ERC guidelines [13] [14]. The mean CPR compression depth showed an adjusted mean depth (SE) of $24.61 \mathrm{~mm}(0.99)$ in the group with CPR feedback, and an adjusted mean (SE) of $20.08 \mathrm{~mm}$ (0.96) in the group without CPR feedback. A box plot of the mean CPR compression depths for the test population is shown in Fig. 3.

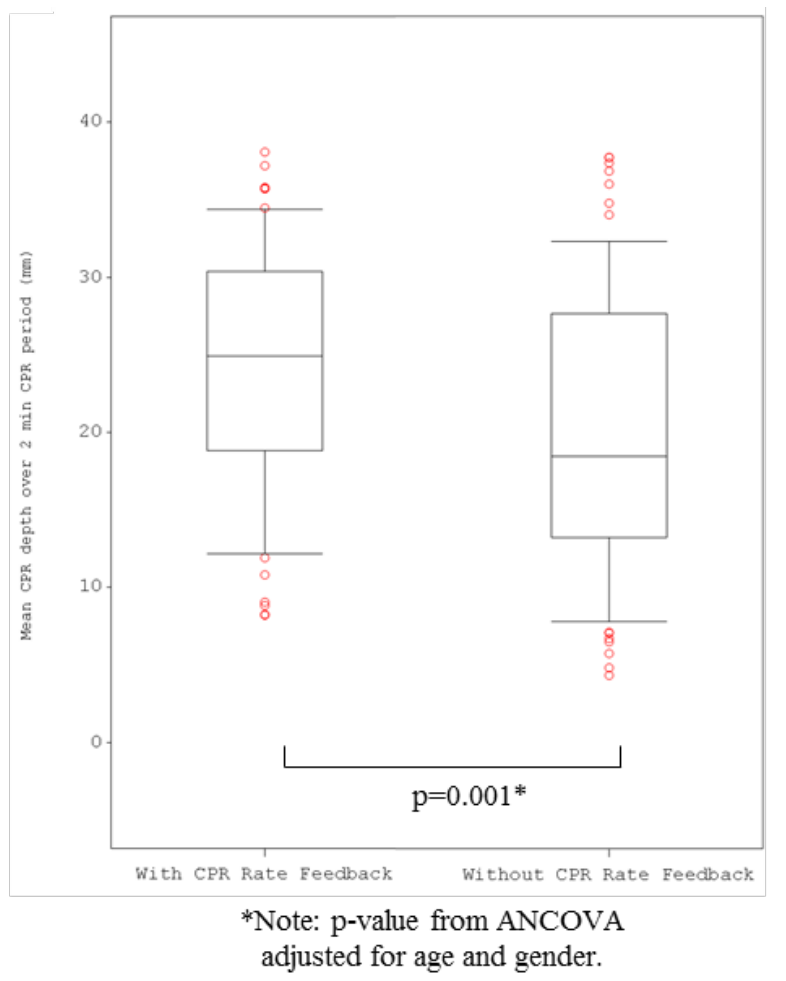

Fig. 3. Box plots showing the mean CPR compression depths (mm) for the devices with and without CPR rate feedback from ANCOVA analysis adjusted for age and gender

The mean percentage CCF (SD) was calculated for both device groups. A CCF of $91.6 \%( \pm 1.4 \%)$ for the group with CPR feedback, and only 1 (1.5\%) participant had a CCF of less than $80 \%$. A mean CCF of $88.7 \%( \pm 1.3 \%)$ was calculated for the group without CPR feedback, and 4 (5.6\%) participants had a CCF of less than $80 \%$. ANCOVA analysis of the mean percentage $\mathrm{CCF}$, provided a mean difference (SE) of $2.9 \%$ $( \pm 1.9 \% ; 95 \% \mathrm{CI}=(-0.9,6.7))$ for the device with $\mathrm{CPR}$ feedback compared to the device without CPR feedback. These CCF data are similar to that of Experiment 1 (CCF $90.3 \%$ ).

As before, the proportion of participants achieving good CPR compression speed within 45 seconds was calculated. A total of 65 of the 68 participants (95\% CI, 87.6\%, 99.1\%) achieved "Good speed" within 45 seconds of beginning CPR for the test device, and 45 of 72 (95\% CI, 50.3\%, 73.6\%) participants achieved "Good speed" within 45 seconds for the 
control device. This difference was found to be statistically significant (Chi-squared statistic $=22.74, \mathrm{df}=1, p<0.0001$ ).

Ability to achieve good compression speed within 45 seconds was not deemed to be related to previous CPR training, as $78.3 \%$ of trained users achieved good compression speed compared to $78.5 \%$ of untrained users.

Of the 65 participants who achieved "Good speed" with the test device, only 3 participants were unable to maintain that rate throughout the CPR cycle. Similarly, of the 45 participants who achieved "Good speed" with the control device, only 1 participant was unable to maintain the rate.

Seven $(10.3 \%)$ of the total 68 participants in the test device group were prompted to "Push faster", and only $1(1.5 \%)$ participant was prompted to "Push slower". All participants responded positively to these audio-visual prompts.

These findings demonstrate the benefit of the CPR-rate feedback in guiding users to achieve good CPR compression speed in accordance with current international guidelines, and where CPR-rate feedback guides users to an optimal speed, it also helps to maintain compression at this rate.

\section{Discussion}

The key findings of this study suggest that the audio prompts issued by the user interface during CPR are sufficient to improve the rate of compression when a good rate of compression (i.e. a rate below $100 \mathrm{CPM}$ ) had not previously been achieved. In addition, compression depth was not compromised by the addition of the CPR rate feedback.

The effects of compression depth in the presence or absence of CPR rate feedback was studied in Experiment 2, and showed a statistically significant mean difference (SE) in CPR compression depth of $4.52 \mathrm{~mm}( \pm 1.38)$ in favour of the test device (two-sided $p$-value $=0.001$ ). This suggests that CPR rate feedback does not detrimentally affect CPR compression depth, but may contribute to improving compression depths. Although statistically significant, it must be acknowledged that this difference in compression depth is not clinically significant. It is also worth noting that no participants in this study successfully achieved optimal compression depth as recommended by ERC and AHA guidelines [13] [14].

The importance of delivering sufficient chest compression rate and depths for successful resuscitation attempts has been previously highlighted [15] [16]. Despite efforts to ensure that optimal CPR compression rate is achieved during resuscitation attempts, the perceived quality of CPR is often underestimated by the rescuer, both in terms of chest compression rate and depth in the absence of real-time CPR feedback [17]. Cheng et al. showed that rescuers overestimate adequacy of compression rate in the absence of real-time CPR feedback, and that the inclusion of a real-time CPR feedback device may enhance the chest compression speed [17]. A randomised resuscitation study by Fischer et al. compared devices with enabled or disabled audio-visual feedback and found that the feedback-group delivered compression rates closest to the recommended guidelines [18]. This is confirmed in this study, as of the 224 participants who used the devices with CPR rate feedback in Experiment 1 and Experiment 2, a total of 201
(89.7\%) achieved compression rates in line with international resuscitation guidelines.

A prospective in-hospital review of compression rates recorded during cardiac arrest resuscitations by Abella et al. investigated the quality of CPR (specifically chest compression rates) performed by trained healthcare providers. Moreover, they found that poor compression rates correlated with decreased return of spontaneous circulation (ROSC) [19].

The average CCF for those participants who received CPR rate feedback in Experiments 1 and 2 was over 90\%. The CPR-rate feedback incorporated in this device was in line with the 2010 American Heart Association and European Resuscitation Council guidelines [13] [14] which state that CCF should be over $80 \%$. Only 16 participants in Experiment 1 and 6 participants in Experiment 2 were unable to achieve guideline $\mathrm{CCF}$.

It must be acknowledged that the depths achieved in Experiment 2 were not in line with the international compression guidelines. However, the aim of this study was not to coach users to perform guideline depth CPR and no compression depth feedback was given. Over half (57.1\%) of all the participants in Experiment 2 were untrained which may also be a reason for below guideline compression depth. Although 60 participants $(42.9 \%)$ had previously received CPR training, lay person CPR skill retention rates are reported to decrease in as little as two months [10], which may lead to inadequate compression depths. Future studies are warranted to assess the ability of a lay-user to achieve guideline compression depth with the assistance of CPR depth feedback.

Time-to-first-shock increased with increasing age groups, 65,67 and 71 seconds respectively. The times collected were compared to a reference time determined by Andre et al., who conducted a study involving 64 participants who engaged with four different AEDs [20]. Andre et al. calculated a weighted mean time-to-first-shock, which was 122 seconds across the four defibrillators investigated. All participants in this study delivered a shock in less than the 122 second reference time, so it can be concluded that the user interface being assessed achieved superiority compared to this reference value.

Although the reference time-to-first-shock (122 seconds) determined by Andre et al. was based on a different population, most variables and study conditions are similar to these experiments, and are not expected to have affected the comparison. Both studies reported on similar variables - the participant's ability to successfully switch the device on, acceptability of electrode placement, time-to-first-shock, and safety during shock delivery. As Andre et al. recruited volunteers who were untrained in CPR-D, it should be noted that the subset of unexperienced participants in our study also provided a median time-to-first-shock significantly below the reference time. Andre et al. recruited a considerably smaller test set $(n=64)$ compared to this study and did not report full demographic information which does not enable a precise comparison of study populations.

Additionally, in a study by Eames et al., 24 untrained participants were asked to perform simulated resuscitation [21], similar to this study. The average time taken reported by 
Eames et al. to deliver a shock was 103.7 seconds, considerably higher than this study.

The study presented herein only assessed a "one rescuer" resuscitation scenario. In a PAD resuscitation event, it is unlikely that a single person will be responsible for the entire event. An additional person to assist with compressions may be a means of ensuring that compression depth is not compromised. Of note, in a study conducted on teams of three trained medical professionals, the median time taken to deliver a first shock was considerably higher than in the study enclosed (100 seconds compared to 67 seconds) [22].

Although every effort was made to ensure that the participant would act as if it were an emergency, we cannot validate if they did so. Thus, the results may have been affected either positively or negatively. In addition, effective CPR requires the rescuer to follow the correct compression rate, provide sufficient compression depth and minimise hands-off time, but also incorporate ventilations and the effects of chest recoil/rescuer leaning contributing to a CPR protocol which maximises survival. Using this type of study model did not assess the effects of these parameters and will require further consideration in future studies.

\section{CONCLUSION}

The incorporation of an embedded computer program in public access defibrillators for providing automated audiovisual feedback can assist trained and untrained personnel to improve chest compression rates and maintain a high CCF as advocated in the resuscitation guidelines. AEDs are expected to become widely available to the minimally trained and untrained user. Therefore, usability, with a particular focus on CPR feedback, must be enhanced to continue improving outcomes from sudden cardiac arrest.

\section{REFERENCES}

[1] Atwood C., Eisenberg M.S., Herlitz J., Rea T.D. Incidence of EMS-treated out-of-hospital cardiac arrest in Europe. Resuscitation 2005;67:75-80

[2] Go A.S., Mozaffarian D., Roger V.L. et al. Heart disease and stroke statistics - 2014 update: a report from the American Heart Association. Circulation 2014;129:e28e292

[3] Handley A.J., Koster R., Monsieurs K., Perkins G.D., Davies S., Bossaert L. European Resuscitation Council Guidelines for Resuscitation 2005. Section 2. Adult basic life support and use of automated external defibrillators. Resuscitation 2005;67S1:7-23

[4] Nichol G., Stiell I.G., Laupacis A., Pham B., De Maio V.J., Wells G.A. A cumulative meta-analysis of the effectiveness of defibrillator-capable emergency medical services for victims of out-of-hospital cardiac arrest. Ann Emerg Med 1999;34:517-525

[5] Hallstrom A.P., Ornato J.P., Weisfeldt M. Public-access defibrillation and survival after out-of-hospital cardiac arrest. N Engl J Med $2004 ; 351(7): 637-646$

[6] Sunde K., Wik L., Naess P.A., Grund F., Nicolaysen G.,. Steen P.A. Improved haemodynamics with increased compression-decompression rates during ACD-CPR in pigs. Resuscitation 1998;39:197-205

[7] Meaney P.A., Bobrow B.J., Mancini M.E. et al. CPR Quality: Improving Cardiac Resuscitation Outcomes both Inside and Outside the Hospital: A Consensus Statement from the American Heart Association. Circulation 2013; 128:417-435.

[8] Yang Z., Li H, Yu T, et al. Quality of chest compressions during compression-only CPR: a comparative analysis following the 2005 and 2010 American Heart Association guidelines. Am Jour of Emerg Med 2014; 32:50-54.

[9] Wik L., Kramer-Johansen J., Myklebust H. et al. Quality of cardiopulmonary resuscitation during out-of-hospital cardiac arrest. Jour Am Med Assoc 2005;293: 299-304.

[10] Yeung J, Okamoto D, Soar J, Perkins D. AED training and its impact on skill acquisition, retention and performance.A systematic review of alternative training. Resuscitation 2011;82(6):657-664

[11] Wik L, Myklebust H, Auestad BH, Steen PA. Twelvemonth retention of CPR skills with automatic correcting verbal feedback. Resuscitation 2005;66:27-30

[12] Kalbfleisch, J. D. and Prentice, R. L. (1980), The Statistical Analysis of Failure Time Data, New York: John Wiley \& Sons.

[13] Nolan J., Soar J.P., Zideman D.A., et al. European Resuscitation Council Guidelines for Resuscitation 2010 Section 1. Executive summary. Resuscitation 2010;18:1219-1276

[14] Berg R.A., Hemphill R., Abella B.S., et al. Part 5: adult basic life support: 2010 American Heart Association Guidelines for Cardiopulmonary Resuscitation and Emergency Cardiovascular Care. Circulation 2010;122 (18 Suppl 3):S685-S705

[15] Morrison LJ, Deakin CD, Morley PT et al. Part 8: Advanced life support: 2010 International Consensus on Cardiopulmonary Resuscitation and Emergency Cardiovascular Care Science with Treatment Recommendations. Circulation 2010; 122:s345-s421

[16] Koster RW, Baubin MA, Bossaert LL, et al. European Resuscitation Council Guidelines for Resuscitation 2010 Section 2. Adult basic life support and use of automated external defibrillators. Resuscitation 2010;81:1277-1292.

[17] Cheng A., Overly F., Kessler D., Nadkarni VM. et al. Perception of CPR quality: Influence of CPR feedback, Just-in-Time CPR training and provider role. Resuscitation 2015;87:44-50.

[18] Fischer H., Gruber J., Neuhold S et al. Effects and limitations of an AED with audio-visual feedback for cardiopulmonary resuscitation: A randomized manikin study. Resuscitation 2011;82:902-907

[19] Abella B.S., Alvarado J.P., Myklebust H. et al. Quality of cardiopulmonary resuscitation during in-hospital cardiac arrest. J Am Med Assoc, 2005;293:305-

[20] Andre A.D., Jorgenson D.B., Froman J., Snyder D.E., Poole J.E. Automated external defibrillator use by untrained bystanders: Can the public-use model work? 
Prehosp Emerg Care 2004;8:284-291.

[21] Eames P., Larsen P.D., Galletly D.C., Comparison of Ease of User of Three Automated External Defibrillators by Untrained Lay People. Resuscitation 2003;58:25-30

[22] Marsch S., Tchan F., Semmer N. et al. Performance of first responders in simulated cardiac arrests. Critical Care Medicine 2005;33.5:963-967 